\title{
Longitudinal dispersion with time-dependent source concentration in semi-infinite aquifer
}

\author{
Mritunjay Kumar Singh*, Nav Kumar Mahato and Premlata Singh \\ Department of Applied Mathematics, Indian School of Mines University, Dhanbad 826 004, India. \\ *e-mail: drmks29@rediffmail.com
}

\begin{abstract}
An analytical solution is obtained to predict the contaminant concentration along unsteady groundwater flow in semi-infinite aquifer. Initially, the aquifer is not supposed to be solute free, i.e., aquifer is not clean. A time-dependent source concentration is considered at the origin of the aquifer and at the other end of the aquifer, it is supposed to be zero. The time-dependent forms of unsteady velocities are considered in which one such form, i.e., sinusoidal form represents the seasonal pattern in a year in tropical regions. The Laplace Transformation Technique (LTT) is used to get an analytical solution and a graphical representation is made through MATLAB.
\end{abstract}

\section{Introduction}

Contamination problems in the groundwater system is a serious concern these days because a large number of toxic compounds are utilized in various industries, especially coal-based industries in the industrial states such as Jharkhand and its neighbouring states. These industries discharge their waste into the neighbouring ponds, streams, rivers, etc. The chemical constituents of the waste material such as expired medicines, pesticides, fertilizers, mine spoils, and those from cemeteries, septic tanks, etc., often infiltrate from the ponds into the groundwater system. The movement of these contaminants through soil to the groundwater system is affected by many factors including the properties of contaminants itself. The transport and fate of contaminants in the subsurface have been very well reported in USEPA (1989). Basically, there are two processes such as dispersion and mass flow by which contaminants move from the earth surface to soil and groundwater. Both these processes are affected by the properties of the contaminants, the soil, the intermediate vadose zone and the aquifer, climatological factor and vegetation pattern, etc. The groundwater contamination is normally traced back to four main sources such as industrial, domestic, agricultural and environmental contamination by Sharma and Reddy (2004). The dispersion sources in uniform groundwater flow have been reported by Hunt (1978). General theory of dispersion in porous media has been very well compiled by Scheidegger (1961).

Groundwater contamination is a problem which affects every individual. In India, most of the people residing in slum areas are bound to use contaminated water because they do not even know the consequences of contaminated water. The present study will certainly enable awareness among people regarding how we are contaminating directly or indirectly, precious natural resources, i.e., groundwater resources and our efforts to rehabilitate contaminated aquifer. Hydrologists, Civil Engineers, Scientists, Professionals, and Academicians are doing their best to solve this type of serious problem by various means. Mathematical model is one of the powerful tools to project the existing problems and its appropriate solutions. As groundwater tends to move very slowly ranging from $2 \mathrm{~m} / \mathrm{yr}(0.005 \mathrm{~m} /$ day $)$ to $2 \mathrm{~m} /$ day, a long time period may elapse after the start of contamination, before affected water shows up in a well. For the same reason, many years may be needed to rehabilitate the contaminated aquifers

Keywords. Dispersion; groundwater contamination; aquifer. 
after the source of contamination has been eliminated. As soon as the contaminants reach the groundwater table, they tend to move along the groundwater flow and the concentration of contaminants decreases due to hydrodynamic dispersion and other attenuation effects like adsorption, first order chemical reaction, radioactive decay, etc. Groundwater transport and its mathematical models were presented by Fried (1975) and Javendal and Tsang (1984). Likewise, analytical solutions of the solute transport equation with rate-limited desorption and decay were presented by Fry et al (1993). Van Genuchten and Alves (1982) presented analytical solutions of one-dimensional convective dispersive solute transport equation under a variety of conditions. Kumar (1983) presented time dependent input concentration against exponentially decreasing unsteady velocity. In the present work, time-dependent forms of input concentration are considered with exponentially decreasing unsteady velocity but not the same as it was proposed by Kumar (1983). In most investigations, groundwater velocity has been considered steady. However, when the groundwater table rises and falls, the velocity of flow in the aquifer may be transient or unsteady. The objective of this study is to derive an analytical solution for space-time distribution of the concentration of contaminants in a semi-infinite aquifer in which the flow velocity is unsteady, and the aquifer is subjected to timedependent point-source contamination.

\section{Mathematical formulation of the problem}

A homogeneous semi-infinite aquifer which may be clean or may have an initial natural concentration is considered. The aquifer is subjected to contamination through a time-dependent source of concentration. The groundwater flow in the aquifer is unsteady where the velocity follows either a sinusoidal form or an exponentially decreasing form. The sinusoidal form of velocity may represent the seasonal variation in a year in tropical regions.

In order to mathematically formulate the problem, let $c(x, t)$ be the concentration of contaminants in the aquifer $\left[\mathrm{ML}^{-3}\right], u$ the groundwater velocity $\left[\mathrm{LT}^{-1}\right]$, and $D$ the dispersion coefficient $\left[\mathrm{L}^{2} \mathrm{~T}^{-1}\right]$ at any time $t[\mathrm{~T}]$. Then the problem can be mathematically formulated as follows:

$$
\begin{aligned}
& D \frac{\partial^{2} c}{\partial x^{2}}-u \frac{\partial c}{\partial x}=\frac{\partial c}{\partial t}, \\
& u(t)=u_{0} V(t),
\end{aligned}
$$

where $u_{0}$ is the initial ground water velocity $\left[\mathrm{LT}^{-1}\right]$ at distance $x[\mathrm{~L}]$.

Here, two forms of $V(t)$ are considered such as $V(t)=1-\sin m t$ and $V(t)=\exp (-m t), m t<1$ where $m$ is the flow resistance coefficient $\left[\mathrm{T}^{-1}\right]$. The dispersion coefficient varies approximately in direct proportion to the seepage velocity for various types of porous media (Ebach and White 1958). It has also been found that such a relationship established for steady flow is also approximately valid for unsteady flow with sinusoidal varying seepage velocity (Rumer 1962). Let $D=a u$, where $a$ is the coefficient of dimension length, depending upon the pore system geometry and the average pore size diameter of porous medium.

Let $c_{i}$ be the initial concentration $\left[\mathrm{ML}^{-3}\right]$ describing the distribution of the concentration at all points of the flow domain at the beginning of investigation, i.e., at $t=0$. It is assumed that initially, the aquifer is not clean. The timedependent input concentration of contaminants in the aquifer is considered at the origin, i.e., $x=0$ and let the concentration at infinite extent be supposed to be zero at all time. Here, the concentration is prescribed for all points of the boundary for the entire period of investigation. The boundary conditions describe the nature of interaction of the flow system with its surrounding. Hence, the initial and boundary conditions can be expressed as:

$$
\begin{aligned}
c(x, t) & =c_{i} ; \quad x \geq 0, t=0, \\
c(x, t) & =c_{0}[1+\exp (-q t)] ; \quad x=0, t \geq 0 \\
& =0 ; \quad t \geq 0, x \rightarrow \infty
\end{aligned}
$$

where $c_{0}$ is the solute concentration $\left[\mathrm{ML}^{-3}\right]$ and $q$ is the parameter $\left[\mathrm{T}^{-1}\right]$ like a flow resistance coefficient.

\section{Analytical solution}

Using equation (2), we get $D=D_{0} V(t)$. Here $D_{0}=a u_{0}$ is an initial dispersion coefficient. Equation (1) can now be written as

$$
D_{0} \frac{\partial^{2} c}{\partial x^{2}}-u_{0} \frac{\partial c}{\partial x}=\frac{1}{V(t)} \frac{\partial c}{\partial t}
$$


A new time variable (Crank 1975), is introduced by the transformation

$$
T^{*}=\int_{0}^{t} V(t) d t
$$

Equation (5) becomes:

$$
D_{0} \frac{\partial^{2} c}{\partial x^{2}}-u_{0} \frac{\partial c}{\partial x}=\frac{\partial c}{\partial T^{*}} .
$$

The initial condition (3) and boundary conditions (4a) and (4b) become:

$$
\begin{aligned}
c\left(x, T^{*}\right) & =c_{i} ; \quad T^{*}=0, x \geq 0, \\
c\left(x, T^{*}\right) & =c_{0}\left(2-q T^{*}\right) ; \quad x=0, T^{*} \geq 0 \\
& =0 ; \quad T^{*} \geq 0, x \rightarrow \infty .
\end{aligned}
$$

Now, the following nondimensional variables are introduced:

$$
\begin{aligned}
& C=\frac{c}{c_{0}}, \quad X=\frac{x u_{0}}{D_{0}}, \\
& T=\frac{u_{0}^{2} T^{*}}{D_{0}}, \quad Q=\frac{q D_{0}}{u_{0}^{2}} .
\end{aligned}
$$

As a result the problem becomes:

$$
\begin{gathered}
\frac{\partial^{2} C}{\partial X^{2}}-\frac{\partial C}{\partial X}=\frac{\partial C}{\partial T} . \\
C(X, T)=\frac{c_{i}}{c_{0}} ; \quad X \geq 0, T=0 . \\
C(X, T)=2-Q T ; \quad X=0, T \geq 0, \\
=0 ; \quad T \geq 0, X \rightarrow \infty .
\end{gathered}
$$

Using the transformation:

$$
C(X, T)=K(X, T) \exp \left(\frac{X}{2}-\frac{T}{4}\right)
$$

in equations (11)-(13) and applying Laplace transformation technique, we may get the solution of the problem as follows:

$$
C(X, T)=\frac{1}{2}\left(2-\frac{c_{i}}{c_{0}}\right)\left[\operatorname{erfc}\left(\frac{X}{2 \sqrt{T}}-\frac{\sqrt{T}}{2}\right)\right.
$$

$$
\left.+\mathrm{e}^{X} \operatorname{erfc}\left(\frac{X}{2 \sqrt{T}}+\frac{\sqrt{T}}{2}\right)\right]+\frac{c_{i}}{c_{0}}
$$$$
-\frac{Q}{2}\left[(T-X) \operatorname{erfc}\left(\frac{X}{2 \sqrt{T}}-\frac{\sqrt{T}}{2}\right)\right.
$$$$
\left.+(T+X) \operatorname{erfc}\left(\frac{X}{2 \sqrt{T}}+\frac{\sqrt{T}}{2}\right)\right] .
$$

\section{An example application and discussion}

Banks and Jerasate (1962) presented the problem of unsteady flow in porous medium. Longitudinal dispersion in such an unsteady flow, derived linear and exponentially time-dependent forms of seepage velocity. In the present discussion, sinusoidal and exponential forms of velocity expressions are considered as follows:

$$
\begin{aligned}
& u(t)=u_{0}(1-\sin m t), \\
& u(t)=u_{0} \exp (-m t), \quad m t<1,
\end{aligned}
$$

where $m$ (/day) is the flow resistance coefficient. The former can very well represent the seasonal behaviour of sinusoidal nature in groundwater velocity and level in aquifers of tropical regions. In such regions, groundwater velocity and level are minimum during the peak of the summer season (the period of greatest pumping) which falls in the month of June, just before the rainy season. Also, both have maximum values during the peak of winter season around the month of December, after the rainy season (the period of lowest pumping out). In tropical regions, the main source of replenishment of groundwater is infiltration from rainfall and from rivers in spate. 


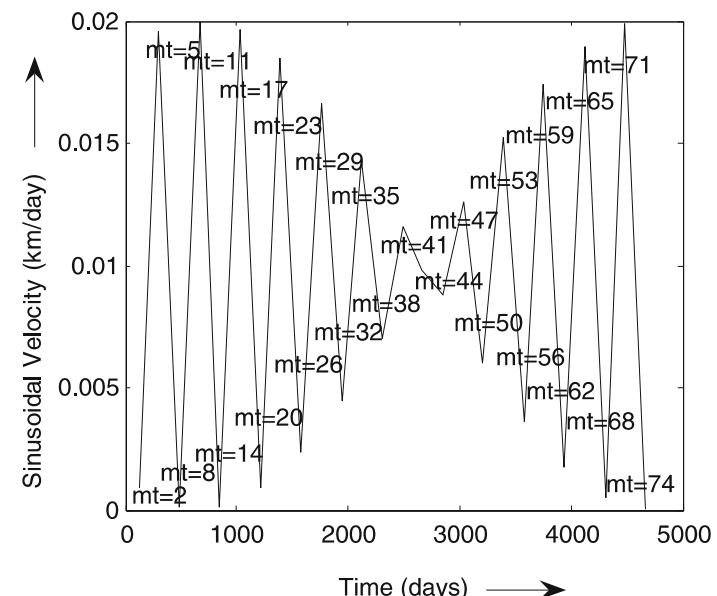

Figure 1. Time-dependent sinusoidal form of velocity representations.

For both the expressions, the non-dimensional time variable $T$ can be written as follows:

$$
\begin{aligned}
& T=\frac{u_{0}^{2}}{m D_{0}}[m t-(1-\cos m t)], \\
& T=\frac{u_{0}^{2}}{m D_{0}}[1-\exp (-m t)] .
\end{aligned}
$$

Now $m t=2,5,8,11,14,17, \ldots, 62,65,68,71$, $74,77,80$ are chosen. For $m=0.0165$ (/day), equation (16a) yields, $t$ (days) $=121.2,303.0$, $484.8,666.6,848.5,1030.3, \ldots, 3757.5,3939.3$, 4121.2, 4303.0, 4484.8, 4666, and 4848.4, respectively. For these values of $m t$, the velocity $u$, is alternatively minimum and maximum. Hence, it represents the groundwater level and velocity minimum during the month of June and maximum during December just after six months in one year. The next data of $t$ represents minimum and maximum records during June and December respectively, in the subsequent years. These representations have been made in figure 1. Analytical solution (15) is solved for the values $c_{i}=0.1$, and $c_{0}=1.0, u_{0}=0.01 \mathrm{~km} /$ day, $D_{0}=0.1 \mathrm{~km}^{2} /$ day, $q=0.0001$ (/day), $x=100 \mathrm{~km}$. The concentration values are depicted graphically in the presence of time-dependent source of contaminants at $m t=26,29,32,35,38$, and 41 which represent minimum and maximum records of groundwater level and velocity during June and December in 5th, 6th and 7th years, respectively. Analytical solution of concentration distribution behaviour of contaminants along the unsteady flow of sinusoidal form given by equation (16a) is represented by the equation (15) and depicted
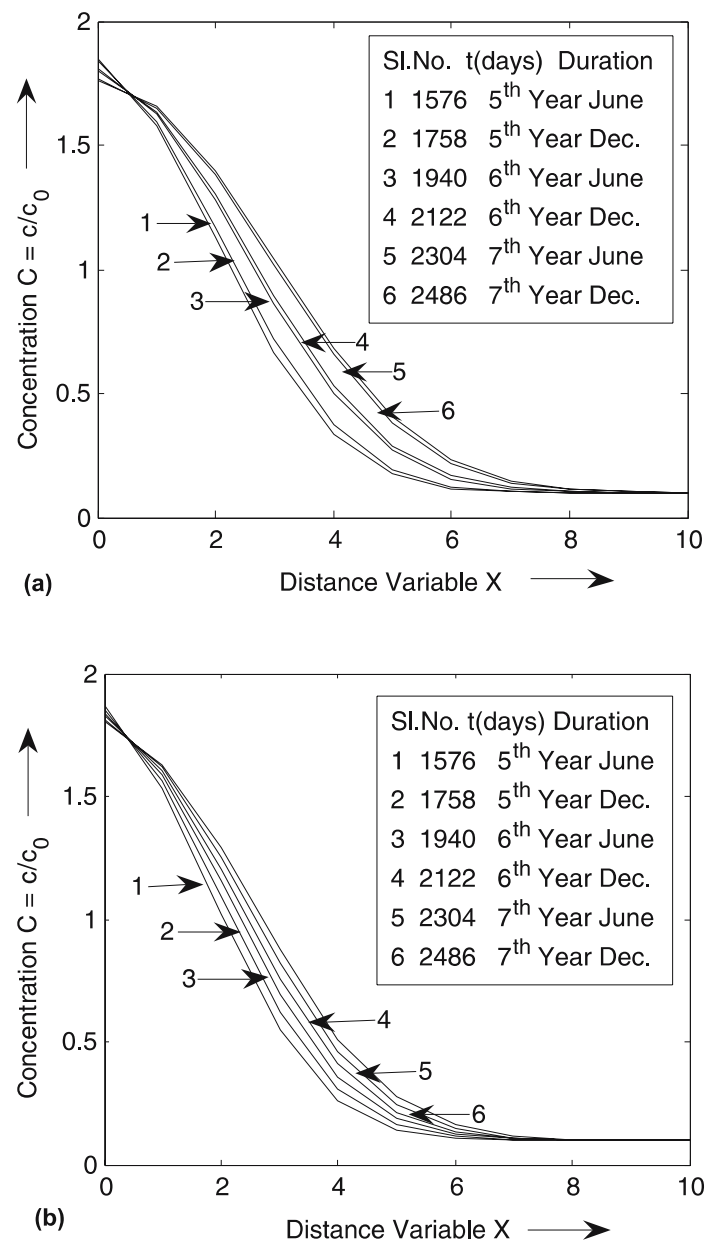

Figure 2. Time-dependent source concentrations along (a) sinusoidal form of velocity and (b) exponentially decreasing form of velocity in semi-infinite aquifer. The line nos. 1-2, 3-4, and 5-6 represent the contaminant concentrations in the 5 th year, 6th year, and 7 th year of June and December respectively.

graphically in figure $2(\mathrm{a})$. For the same set of inputs except $m=0.0002$ (/day) as $m t<1$, equation (15) is also computed for exponentially decreasing form of velocity given by equation (16b), and depicted graphically in figure 2(b). It is observed that the source concentration at the origin of the aquifer is decreasing with time for both the unsteady velocities and near by the origin, it starts slightly decreasing and emerging at the common point. It is also observed that after emergence, it goes on decreasing for sinusoidal form of velocity as well as exponential form of velocity. The rate of decreasing tendency for sinusoidal form of velocity is slightly slower than the exponential form of velocity. In both cases, the decreasing tendency of the concentration levels of contaminants with time and distance travelled, may help to rehabilitate the contaminated aquifer and may be useful for groundwater resource management. 


\section{Conclusion}

The concentration distribution behaviour of contaminants is depicted by an analytical solution of homogeneous semi-infinite aquifer along unsteady groundwater flow with time-dependent source of contaminants. Initially aquifer is not solute free. The solutions are obtained for sinusoidal form of velocity which represent the seasonal pattern in tropical regions and the exponential form of velocity too. The output of the problems may help to know the position and the time period of harmless concentration levels of the contaminated aquifer. The solution may be used as a preliminary predictive tool for simulating the contaminant migration in aquifer due to the release of a time-dependent source.

\section{Acknowledgements}

Authors are grateful to Dr M Mojibur Rahman, Assistant Professor, Department of Humanities and Social Science, Indian School of Mines University, Dhanbad, for his co-operation in editing this work. Authors are thankful to the reviewers for their valuable suggestions and modifications incorporated in the manuscript.

\section{References}

Banks R P and Jerasate S T 1962 Dispersion in unsteady porous media flow; J. Hydraulic. Div. 88121 p.

Crank J 1975 The Mathematics of Diffusion (Oxford: Oxford University Press).

Ebach E H and White R 1958 Mixing of fluid flowing through beds of packed solids; J. Am. Inst. Chem. Eng. 4 161-164.

Fried J J 1975 Groundwater Pollution (Amsterdam: Elsevier Scientific Publishing Company).

Fry V A, Istok J D and Guenther R B 1993 An analytical solution of the solute transport equation with ratelimited desorption and decay; Water Resource Res. 29(9) 3201-3208.

Hunt B 1978 Dispersion sources in uniform ground water flow; J. Hydraulic Div. 104(1) 75-85.

Javendel I C and Tsang C F 1984 Groundwater transport: Handbook of mathematics models; Water Resource Monogrser 10 (AGU Washington).

Kumar N 1983 Unsteady flow against dispersion in finite porous media; J. Hydrol. 63 345-358.

Rumer R R 1962 Longitudinal dispersion in steady and unsteady flow; J. Hydraulic Div. 88(HY4) 147-172.

Scheidegger A E 1961 General theory of dispersion in porous media; J. Geophys. Res. 6610 p.

Sharma H D and Reddy K R 2004 Geo-environmental Engineering (John Wiley \& Sons, Inc.).

USEPA 1989 Transport and Fate of Contaminants in the Subsurface; EPA 625(19) 4-89 (USEPA, Washington, DC).

Van Genucheten M Th and Alves W J 1982 Analytical solution of one dimensional convective-dispersion solute transport equation; Tech. Bull. 1661151 p. 